Dorota Kozakiewicz-Kłosowska

Università di Varsavia

https://doi.org/10.18778/8220-506-0.03

\title{
L'ITALIANITÀ E LA POLONITÀ NEL CONCETTO DELLA SOCIETÀ CIVILE
}

\begin{abstract}
Riassunto: Il soggetto dell'analisi sono gli esponenti lessicali del concetto "società civile" e "responsabilità sociale" / "senso civico" tratti dal corpus di testi delle pubblicità sociali italiane e polacche (1972-2017). Il termine "società civile" significa in generale il coinvolgimento di ogni persona nella costruzione di condizioni di vita favorevoli all'interno di una data realtà sociale. Si tratta di un atteggiamento caratterizzato dall'attività volontaria dei cittadini, a favore dei cambiamenti in meglio nel loro ambiente sociale. Si basa sul presupposto che ogni individuo abbia un impatto sulla qualità della vita a livello locale e sovra-locale. Il punto di partenza per considerare il concetto della "società civile" è un uomo autonomo, tollerante, aperto e pronto ad agire per il bene degli altri. La relazione si propone di verificare quali esponenti linguistici provenienti dal discorso pubblicitario pro publico bono formano una definizione aperta del concetto preso in considerazione, negli ultimi 50 anni in Polonia e in Italia, secondo una chiave comparativa.
\end{abstract}

Parole chiave: società civile, responsabilità sociale, pubblicità.

Abstract: Italianity and Polonity in the concept of civil society. The subject of the analysis are the lexical exponents of the concept of "civil society" and "social responsibility" taken from the corpus of texts of Italian and Polish social advertising (1972-2017). The term "civil society" generally means the involvement of each person in the construction of propitious living conditions within a given social reality. It is an attitude characterized by the voluntary activity of citizens, in favor of changes for the better in their social environment. It is based on the assumption that each individual has an impact on quality of life at the local and supra-local level. The starting point for considering the concept of "civil society" is an autonomous, tolerant, open unit, ready to act for the good of others. The article aims to verify which linguistic exponents from the pro publico bono advertising form an open definition of the concept taken into consideration, in the last 50 years in Poland and in Italy, in a comparative key.

Keywords: civil society, social responsibility, publicity. 


\section{Introduzione}

In questa sede si vogliono proporre delle semplificate definizioni cognitive dei concetti: "società civile" e "responsabilità sociale" / "senso civico" (pol. społeczeństwo obywatelskie e odpowiedzialność spoteczna) che determinano il significato della "società civile", le quali emergono dal corpus di testi provenienti dal discorso della pubblicità sociale (DPS) italiana e polacca. Si basano sui risultati di una ricerca prima di tutto quantitativa, sostenuta dal software Sketch Engine per l'analisi dei corpora linguistici. Il corpus raccolto è composto di un totale di 141.537 token nella parte italiana, 104.551 token nella parte polacca. Le pubblicità dalle quali sono stati trascritti i testi erano lanciate in Italia e in Polonia tra il 1972 ed il 2017. Si presentano pertanto i componenti linguistici che formano una definizione aperta dei menzionati concetti negli ultimi 50 anni in Polonia e in Italia, secondo una chiave comparativa. Per le caratteristiche che segnano l'italianità e la polonità nei concetti si intendono gli esponenti lessicali (verbali, sostantivali e aggettivali) riflessi nelle parole chiave estratte dal menzionato software, dalla parte italiana e polacca del corpus. Nell'elaborazione delle definizioni si è basati principalmente sulla teoria dei frames di Charles Fillmore per (1) identificare con quali unità lessicali viene schematizzato il concetto "responsabilità sociale" / "senso civico" nel discorso delle pubblicità sociali e (2) creare delle definizioni cognitive, in base ai testi pubblicitari raccolti. I frames rappresentano delle descrizioni (contesti, soprattutto lessicali) legate ai gruppi di lessemi che hanno qualcosa in comune. Il modello concettuale di Fillmore (2006: 373) presuppone, in generale, l'esistenza di elementi tipici per delle situazioni linguistiche specifiche, nonché la presenza dei componenti linguistici indispensabili, affinché un determinato concetto abbia significato e senso. Ogni unità lessicale richiama un sapere contestuale - "scenario" (ingl. semantic frame $e^{1}$ ). Lo schema delle definizioni proposte è fondato su quello della definizione cognitiva. Nella prima parte si presenta brevemente una sintesi delle definizioni enciclopediche dei concetti, in un secondo momento vengono riportate sei tabelle con delle parole chiave (e le rispettive frequenze); vale a dire gli esponenti lessicali del frame analizzato.

1 Cfr. Modello cognitivo. Il concetto del framing fa riferimento alle operazioni del cervello umano, facenti parte del processo di categorizzazione (Croft 2002; Gleason, Ratner 2005). Ė pertanto presente in tutti i processi cognitivi legati all'acquisizione, all'elaborazione e all'interpretazione dei dati dal cervello. Consiste nel richiamare alcuni modelli di schemi dalla memoria semantica attraverso uno stimolo quale una specifica parola o un dato concetto. Il sapere al quale si riferiscono i frames è (tra gli altri $\mathrm{il}$ sapere contestuale, una comune esperienza sociale e culturale (Fillmore 2003). 


\section{Definizione enciclopedica}

I termini "società civile" e "responsabilità sociale" / "senso civico" (odpowiedzialność społeczna e społeczeństwo obywatelskie) sono correlati e affini, ma non sinonimici, in quanto il primo si applica a singole persone, e le loro azioni, mentre il secondo si riferisce alla comunità nel suo insieme, ai comportamenti sui quali un individuo ha un'influenza limitata: soltanto quando agisce in sinergia con gli altri. Secondo le fonti enciclopediche ${ }^{2}$, il termine "società civile" si fonda sul coinvolgimento delle persone nella costruzione di buone condizioni di vita. Il punto di partenza per le considerazioni sul concetto in questione è un uomo pronto ad agire per il bene del suo ambiente, impegnato in ciò che possa essere fonte di un'iniziativa utile. (Załęski 2012: 7-24; Krasnowolski 2014: 3-7). Il concetto attuale prende l'origine dall'ordine descritto dai filosofi seicenteschi e ottocenteschi, i fondatori delle fondamenta della moderna organizzazione della vita sociale nelle strutture statali ${ }^{3}$. L'elementare "attivismo", ossia la partecipazione attiva dei cittadini e delle entità sociali ed economiche alla vita sociale dovrebbe verificarsi a prescindere dall'interferenza dello Stato. La responsabilità sociale si forma prima di tutto attraverso le iniziative di associazione civica, economica e culturale, le attività volontarie che talvolta si oppongono alle istituzioni statali o addirittura, contrastano la politica (Bobbio 1976: 27; Gramsci 1949: 9). Al giorno d'oggi, si parla di una reciproca fiducia che sta alla base della società civile. Innanzitutto, influisce sul miglior funzionamento delle istituzioni e dell'amministrazione (comuni, regioni), l'assistenza sanitaria, servizi pubblici, determina la soddisfazione della vita ed il benessere. La mancanza di fiducia e una scarsa responsabilità sociale portano paura, provocano l'indifferenza verso la sorte, possono provocare comportamenti sfavorevoli al bene degli altri, uno sbagliato uso delle risorse umane e del capitale pubblico.

Come si può presumere il frame analizzato è vasto e allo stesso tempo poco preciso, non facile da definire. Copre potenzialmente tutte le questioni relative alle attività sociali benefiche. Si è decisi di scegliere tre "sotto frames" - gli argomenti selezionati per le definizioni in base al corpus dei testi pubblicitari raccolti. Riguardano tre aree tematiche (1-Tab. 1ab) la risposta al danno

2 Cfr. ad esempio www.treccani.it/enciclopedia/societa-civile_\%28Enciclopedia-delle-scienze-sociali\%29/ [11/11/2020].

3 Non è il momento per citare una varietà di considerazioni filosofiche in materia, ma vale la pena ricordare che il modello di un moderno stato democratico in cui vi sono limiti invalicabili di interferenza da parte del governo e dell'amministrazione nella sfera dei diritti fondamentali e delle libertà civili, con la giusta proporzione tra l'interesse dei gruppi e l'interesse pubblico, fa riferimento al pensiero greco. Al giorno d'oggi si traduce nelle basi di società pluralistica liberal-democratica (Blicharz 2012: 55). 
altrui; il fornire e l'offrire assistenza ai bisognosi ed il volontariato (2-Tab. 2ab) l'uguaglianza e pari diritti a tutti i livelli: tra donne e uomini, degli omosessuali, delle persone di origini e nazionalità diverse, (3-Tab. 3ab) il prendersi cura dell'ambiente comune, compreso l'ambiente naturale e lo spazio sociale. Per ciascuno di questi "sotto-frames, si propongono due definizioni aperte, una polacca e una italiana, in base al numero di occorrenze dei sostantivi, aggettivi e verbi chiave provenienti dal corpus e selezionati dallo Sketch Engine. Nella prima colonna vengono riportati (nell'ordine alfabetico, partendo dalle parole in polacco) le parole che hanno una significativa frequenza $\left(\mathrm{N}^{\circ}\right.$ types $)$ nel corpus ${ }^{4}$.

\section{Definizioni cognitive}

Tab. 1a

\begin{tabular}{|c|c|c|}
\hline Parole chiave & $\begin{array}{l}\text { Freq } \\
-\mathbf{N}^{\circ} \\
\text { types }\end{array}$ & $\begin{array}{l}\text { Definizione PL Odpowiedzialność społeczna } \\
\text { w społeczeństwie obywatelskim to: }\end{array}$ \\
\hline 1 & 2 & 3 \\
\hline $\begin{array}{l}\text { CHRONIĆ } \\
\text { OCHRONA } \\
\text { OPIEKOWAĆ (SIE) } \\
\text { OPIEKA }\end{array}$ & $\begin{array}{l}90 \\
100 \\
6 \\
44\end{array}$ & \multirow{6}{*}{$\begin{array}{l}\text { - DAWANIE innym możliwości rozwoju; poda- } \\
\text { rowanie (DZIECIOM) uśmiechu; } \\
\text { - dawanie poczucia bezpieczeństwa (dzieciom, } \\
\text { osobom potrzebującym); } \\
\text { - dawanie życia (nowego, lepszego); oddawanie } \\
\text { części siebie dla innych (krwi, organów). } \\
\text { - KOCHANIE dzieci, kobiet i okazywanie im } \\
\text { miłości, nie agresja, brak przemocy domowej; } \\
\text { - uznanie takiej samej MIŁOŚCI (pomiędzy oso- } \\
\text { bami heteroseksualnymi i lgbt); te same prawa } \\
\text { w miłości; brak przemocy wobec inności, innej } \\
\text { orientacji seksualnej. } \\
\text { - PAMIĘĆ, PAMIETTANIE o innych (słabszych, } \\
\text { potrzebujących); } \\
\text { - pamiętanie, że przemoc jest zła, to przestępstwo } \\
\text { i pozostawia ślad na całe życie. } \\
\text { - POMOC, konkretne POMAGANIE (1\% po- } \\
\text { datku, sms), które się opłaca i/lub przynosi } \\
\text { satysfakcję, wzmacnia poczucie spełnienia; } \\
\text { - oferowanie i udzielanie pomocy potrzebującym } \\
\text { (ludziom i zwierzętom); }\end{array}$} \\
\hline DZIECKO & 610 & \\
\hline $\begin{array}{l}\text { KOCHAĆ } \\
+ \text { KOCHANY } \\
\text { MIŁOŚĆ }\end{array}$ & $\begin{array}{l}113 \\
44\end{array}$ & \\
\hline $\begin{array}{l}\text { NIEPEENOSPRAW- } \\
\text { NOŚĆ + NIEPEŁ- } \\
\text { NOSPRAWNY }\end{array}$ & 122 & \\
\hline $\begin{array}{l}\text { OBOJEcTNOŚĆ } \\
(+ \text { OBOJĘTNY) }\end{array}$ & 32 & \\
\hline $\begin{array}{l}\text { (OD)DAWAĆ } \\
\text { DAWCA } \\
\text { DAROWAĆ (+ DAR } \\
\text { + DARCZYŃCA) }\end{array}$ & $\begin{array}{l}80 \\
46 \\
12\end{array}$ & \\
\hline
\end{tabular}

4 Si vuole sottolineare che la classificazione delle parole nei tre gruppi è intrinseca. 


\begin{tabular}{|c|c|c|}
\hline 1 & 2 & 3 \\
\hline $\begin{array}{l}\text { PAMIËTAĆ } \\
\text { + PAMIEĆC }\end{array}$ & 130 & \multirow{9}{*}{$\begin{array}{l}\text { szukanie odpowiedniej pomocy, proszenie } \\
\text { o pomoc, nie banie się prosić o pomoc; } \\
\text { dbanie o dzieci poszkodowane, ratowanie } \\
\text { dzieci (bitych, chorych, głodnych, niewido- } \\
\text { mych, upośledzonych); dbanie o rozwój dzieci } \\
\text { potrzebujących. } \\
\text { - REAKCJA, REAGOWANIE - brak OBO- } \\
\text { JĘTNOŚCI na akty niszczenia, wandalizmu } \\
\text { (przystanki); } \\
\text { reagowanie na krzywdę innych (dzieci, kobiet, } \\
\text { osób starszych, zwierząt), na akty przemocy } \\
\text { i agresji; } \\
\text { reagowanie na przestępstwa (np. sprzedaż al- } \\
\text { koholu nieletnim, próba siadania za kierownicą } \\
\text { po spożyciu alkoholu), na korupcję.5 } \\
\text { - WALKA z przemocą, hejtem i wandalizmem; } \\
\text { - walka z uprzedzeniami i niesprawiedliwością } \\
\text { społeczną i agresją. } \\
\text { - szukanie WSPARCIA i pomocy; } \\
\text { - WSPIERANIE osób słabszych, chorych, nie- } \\
\text { pełnosprawnych i potrzebujących; } \\
\text { - wspieranie programów pomocowych (1\%). }\end{array}$} \\
\hline $\begin{array}{l}\text { POMAGAĆ } \\
\text { POMOC }(\mathbf{N Y})\end{array}$ & $\begin{array}{l}135 \\
200\end{array}$ & \\
\hline $\begin{array}{l}\text { PORZUCAĆ } \\
\text { ZOSTAWIĆ }\end{array}$ & 7 & \\
\hline $\begin{array}{l}\text { OPUŚCIĆ } \\
+ \text { OPUSZCZONY }\end{array}$ & 0 & \\
\hline $\begin{array}{l}\text { (U)RATOWAĆ } \\
\text { + RATUNEK } \\
\end{array}$ & 124 & \\
\hline $\begin{array}{l}\text { REAGOWAĆ } \\
+ \text { REAKCJA }\end{array}$ & 150 & \\
\hline $\begin{array}{l}\text { WOLONTARIAT } \\
+ \text { WOLONTARIUSZ } \\
\end{array}$ & 63 & \\
\hline $\begin{array}{l}\text { WSPIERAĆ } \\
+ \text { WSPARCIE } \\
\end{array}$ & 74 & \\
\hline $\begin{array}{l}\text { (ZA/Z)WALCZYĆ } \\
\text { WALKA }\end{array}$ & $\begin{array}{l}44 \\
37\end{array}$ & \\
\hline
\end{tabular}

Fonte: elaborazione propria

Tab. $1 b$

\begin{tabular}{|l|l|l|}
\hline Parole chiave & $\begin{array}{l}\text { Freq } \\
-\mathbf{N}^{\circ} \\
\text { types }\end{array}$ & $\begin{array}{l}\text { Definizione IT La responsabilità sociale/ } \\
\text { senso civico nella società civile è: }\end{array}$ \\
\hline \multicolumn{1}{|c|}{1} & 2 & \multicolumn{1}{c|}{3} \\
\hline $\begin{array}{l}\text { ABBANDONARE } \\
\text { + ABBANDONO } \\
\text { LASCIARE }\end{array}$ & $\begin{array}{l}106+ \\
46\end{array}$ & $\begin{array}{l}\text { - AGIRE e AIUTARE attraverso adozione } \\
\text { a distanza, sms, donazioni; aiutare i bambini del } \\
\text { terzo mondo; }\end{array}$ \\
\hline
\end{tabular}

5 La corruzione viene spesso metaforizzata come una malattia, prevenibile e curabile.

6 Si può notare una frequenza significativamente più alta della coppia verbo + sostantivo nella parte polacca del corpus (oltre 150 occorrenze). Diversi fattori influenzano questo fenomeno. In primo luogo, il verbo reagire pol. reagować nella seconda persona singolare dell'imperativo è diventato uno dei simboli della pubblicità sociale in Polonia (pol. reaguj). Nei messaggi polacchi, si sottolinea spesso il fatto che ognuno ha il diritto (e il dovere) di reagire se nota qualcosa di sbagliato. 
Tab. $1 \mathrm{~b}$ (cont.)

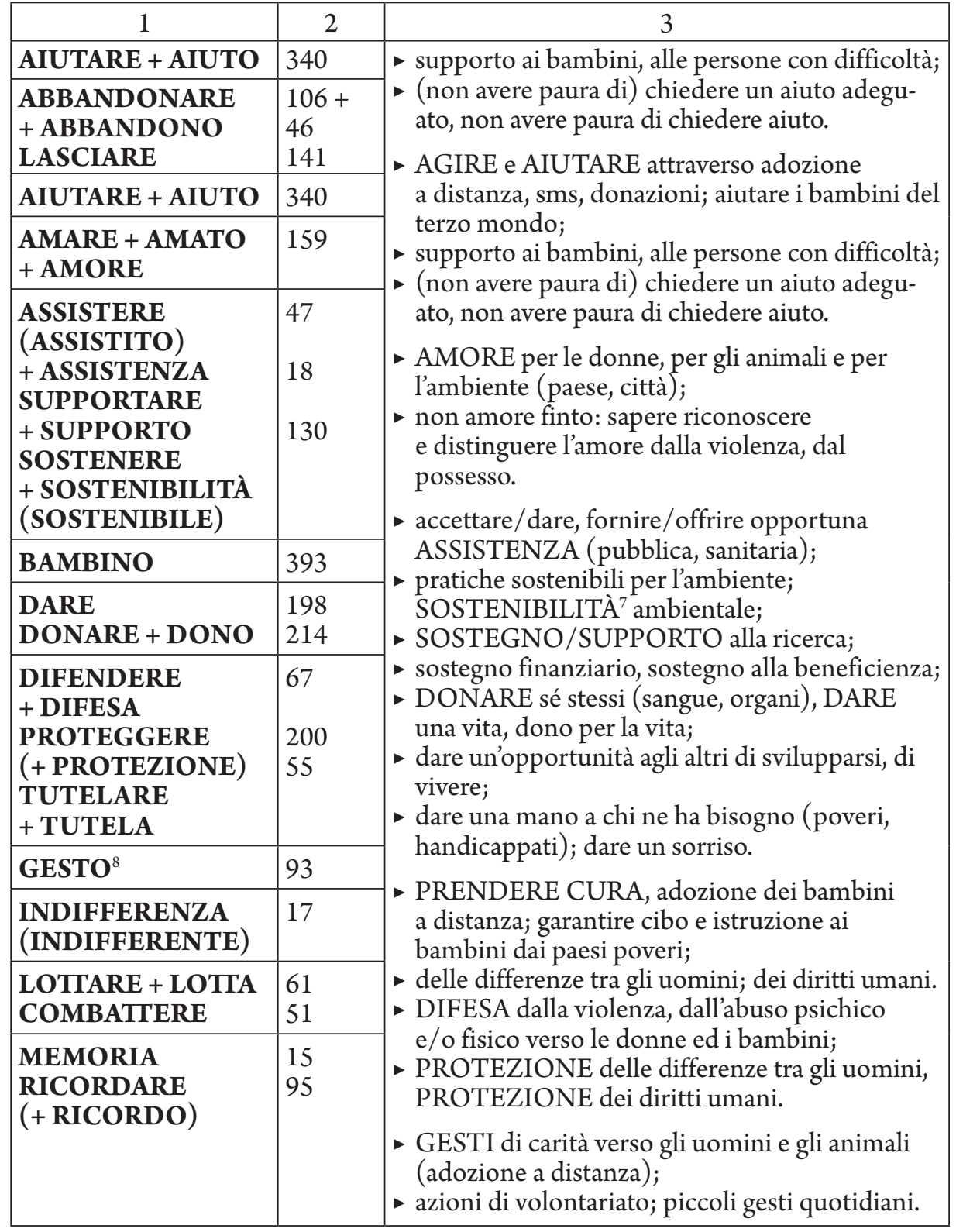

7 Equivalente polacco, zrównoważony (bilanciato) ha solo 5 types in tutto il corpus, nel contesto della protezione ambientale.

8 Gest ha soltanto 5 occorrenze nella parte polacca. 


\begin{tabular}{|c|c|c|}
\hline 1 & 2 & 3 \\
\hline $\begin{array}{l}\text { REAGIRE + } \\
\text { REAZIONE }\end{array}$ & 20 & \multirow{5}{*}{$\begin{array}{l}\text { LOTTA contro i fattori che danneggiano } \\
\text { la salute degli altri (fumo, rumore), che } \\
\text { distruggono l'ambiente naturale; } \\
\text { - lotta contro indifferenza, violenza, abusi } \\
\text { sessuali, bullismo, razzismo, omofobia, } \\
\text { discriminazione. } \\
\text { - LIBERARSI dai pregiudizi (sulle malattie } \\
\text { mentali, razze, sesso); } \\
\text { - NON ABBANDONARE/NON LASCIARE } \\
\text { chi ha bisogno d'aiuto (bambini poveri, gente } \\
\text { povera, animali); } \\
\text { non abbandonare/non lasciare i rifiuti per } \\
\text { strada, in spiaggia, in mare; gli escrementi dei } \\
\text { cani per strada ecc. } \\
\text { RICORDARSI / commemorazione delle } \\
\text { vittime delle mafie (la giornata della } \\
\text { MEMORIA). }\end{array}$} \\
\hline SALVARE & 125 & \\
\hline \multirow[t]{3}{*}{$\begin{array}{l}\text { VOLONTARIATO } \\
\text { + VOLONTARIO }\end{array}$} & 110 & \\
\hline & & \\
\hline & & \\
\hline
\end{tabular}

Fonte: elaborazione propria

Tab. $2 \mathrm{a}$

\begin{tabular}{|c|c|c|}
\hline Parole chiave & $\begin{array}{l}\text { Freq } \\
-\mathbf{N}^{\circ} \\
\text { types }\end{array}$ & $\begin{array}{l}\text { Definizione PL Odpowiedzialność społeczna } \\
\text { w społeczeństwie obywatelskim to: }\end{array}$ \\
\hline 1 & 2 & 3 \\
\hline $\begin{array}{l}\text { DYSKRYMINO- } \\
\text { WAĆ + DYSKRY- } \\
\text { MINACJA (DYS- } \\
\text { KRYMINOWANY) } \\
\text { + WYKLUCZAĆ } \\
\text { + WYKLUCZENIE } \\
\text { (WYKLUCZONY) }\end{array}$ & 50 & \multirow{3}{*}{$\begin{array}{l}\text { - RÓWNOUPRAWNIENIE KOBIET i męż- } \\
\text { czyzn w domu, w pracy; } \\
\text { - RESPEKOTWANIE PRAW i wsparcie dla ko- } \\
\text { biet; } \\
\text { - walka z PRZEMOCĄ wobec kobiet. } \\
\text { niełamanie PRAW człowieka, praw obywatel- } \\
\text { skich; respektowanie i poszanowanie postano- } \\
\text { wień prawa; } \\
\text { prawo do (i obowiązek) głosowania w wybo- } \\
\text { rach; } \\
\text { prawo do godnego życia (bezdomnych, osób } \\
\text { starszych), prawo do pracy (niepełnospraw- } \\
\text { nych); }\end{array}$} \\
\hline $\begin{array}{l}\text { GLOSOWAĆ } \\
+ \text { GŁOS }\end{array}$ & $\begin{array}{l}32 \\
73 \\
\end{array}$ & \\
\hline $\begin{array}{l}\text { KOBIETA } \\
+ \text { KOBIECY }\end{array}$ & 314 & \\
\hline
\end{tabular}


Tab. 2a (cont.)

\begin{tabular}{|c|c|c|}
\hline 1 & 2 & 3 \\
\hline $\begin{array}{l}\text { MĘŻCZYZNA } \\
\text { + MEESKI } \\
\text { CZEOWIEK } \\
\text { + LUDZIE (LUDZKI) }\end{array}$ & & \multirow{6}{*}{$\begin{array}{l}\text { - równe prawa dla wszystkich; } \\
\text { znajomość i świadomość własnych praw. } \\
\text { - równe SZANSE rozwoju dla wszystkich (ko- } \\
\text { biet, mężczyzn, dzieci, niepełnosprawnych); } \\
\text { wyrównanie szans; } \\
\text { - szansa na „,normalne życie”. } \\
\text { - INTEGRACJA osób z niepełnosprawnościami; } \\
\text { zatrudnienie osób NIEPEŁNOSPRAWNYCH, } \\
\text { ułatwianie dostępu do usług (budynków); } \\
\text { pomoc niepełnosprawnym fizycznie i intelektu- } \\
\text { alnie (np. z zespołem Downa). } \\
\text { - NIETOLEROWANIE „złych”, szkodliwych } \\
\text { wobec innych zachowań np. aktów wandalizmu, } \\
\text { zajmowania miejsc niepełnosprawnym na par- } \\
\text { kingach i w komunikacji miejskiej, nieustępo- } \\
\text { wanie miejsca siedzącego osobom uprzywilejo- } \\
\text { wanym; reagowanie na „zle” zachowania; } \\
\text { - oswajanie inności, zwalczanie stereotypów } \\
\text { dotyczących np. osób homoseksualnych, osób } \\
\text { upośledzonych; osób starszych; } \\
\text { - wolontariat, pomoc innym; pomaganie potrze- } \\
\text { bującym (osobom chorym, niepełnosprawnym, } \\
\text { chorym dzieciom, pokrzywdzonym dzieciom). }\end{array}$} \\
\hline PRAWO & 178 & \\
\hline RASA + RASIZM & 5 & \\
\hline $\begin{array}{l}\text { RÓWNOŚĆ + RÓW- } \\
\text { NY + RÓWNO- } \\
\text { UPRAWNIENIE }\end{array}$ & 45 & \\
\hline $\begin{array}{l}\text { SZANSA } \\
\text { MOŻLIWOŚĆ }\end{array}$ & & \\
\hline $\begin{array}{l}\text { TOLEROWAĆ } \\
\text { + TOLERANCJA } \\
\text { (TOLERANCYJNY) }\end{array}$ & 35 & \\
\hline
\end{tabular}

Fonte: elaborazione propria

Tab. 2b

\begin{tabular}{|c|c|c|}
\hline Parole chiave & $\begin{array}{l}\text { Freq } \\
-\mathbf{N}^{\circ} \\
\text { types }\end{array}$ & $\begin{array}{l}\text { Definizione IT La responsabilità sociale/ } \\
\text { senso civico nella società civile è: }\end{array}$ \\
\hline 1 & 2 & 3 \\
\hline $\begin{array}{l}\text { DISCRIMINARE } \\
\text { + DISCRIMINA- } \\
\text { ZIONE (DISCRIMI- } \\
\text { NAO) + ESCLUDE- } \\
\text { RE + ESCLUSIONE } \\
\text { (ESCLUSO) }\end{array}$ & 30 & $\begin{array}{l}\text { avere DIRITTO di sapere, diritto } \\
\text { all'informazione, diritto all'istruzione, diritto } \\
\text { alla formazione; } \\
\text { garanzia dei PARI DIRITTI a tutti (diritti alla } \\
\text { prevenzione, diritti di lavoro per i migranti, } \\
\text { diritto di voto per gli immigrati); }\end{array}$ \\
\hline
\end{tabular}




\begin{tabular}{|c|c|c|}
\hline 1 & 2 & 3 \\
\hline $\begin{array}{l}\text { VOCE } \\
\text { VOTARE + VOTO }\end{array}$ & & \multirow{8}{*}{$\begin{array}{l}\text { non privare dei diritti; } \\
\text { rispettare i diritti dei bambini e donne, degli } \\
\text { animali, dei disabili. } \\
\\
\text { - assenza di discriminazione e PREGIUDIZI } \\
\text { nei confronti con le persone disabili (Down), } \\
\text { essere sensibili ai problemi dei disabili; (re) } \\
\text { inserimento lavorativo degli handicappati; } \\
\text { dare l'OPPORTUNITÀ di integrazione per le } \\
\text { persone con disabilità; } \\
\text { - assenza di discriminazione per razza } \\
\text { o orientamento sessuale; } \\
\text { pari opportunità tra tutti i cittadini; } \\
\text { - PARITA dei sessi tra uomini e donne (parità } \\
\text { donne) nel lavoro, nella politica e a casa; } \\
\text { - POSSIBILITÀ di scelta libera di ogni persona. } \\
\\
\text { - PROTEZIONE DELLE DONNE; FERMARE } \\
\text { la violenza sulle donne, la violenza domestica, i } \\
\text { femminicidio; } \\
\text { - pari opportunità per le donne (a casa, } \\
\text { nel lavoro); la possibilità di esprimersi; } \\
\text { superamento dei pregiudizi nei confronti delle } \\
\text { donne; } \\
\text { - RISPETTO per le donne. }\end{array}$} \\
\hline $\begin{array}{l}\text { DONNA + } \\
\text { FEMMINA } \\
\text { + FEMMINILE }\end{array}$ & 3 & \\
\hline $\begin{array}{l}\text { UOMO + UMAN } \\
+ \text { + MASCHI } \\
\text { ** }\end{array}$ & & \\
\hline $\begin{array}{l}\text { DIRITTO } \\
\text { LEGGE }\end{array}$ & & \\
\hline $\begin{array}{l}\text { RAZZA + } \\
\text { RAZZISMO } \\
\text { (RAZZISTA) }\end{array}$ & 50 & \\
\hline $\begin{array}{l}\text { PARITÀ (PARI) } \\
\text { + UGUAGLIANZA } \\
\text { (UGUALE) }\end{array}$ & 28 & \\
\hline $\begin{array}{l}\text { POSSIBILITÀ } \\
\text { OPPORTUNITÀ }\end{array}$ & & \\
\hline $\begin{array}{l}\text { TOLLERARE } \\
+(\text { IN)TOLLERAN- } \\
\text { ZA }\end{array}$ & $<20$ & \\
\hline
\end{tabular}

Fonte: elaborazione propria

È opportuno notare che in entrambele parti del corpus, i sostantivi "kobieta" e "donna/femmina", e i loro derivati, hanno una frequenza particolarmente. La maggior parte delle occorrenze si verifica nel contesto della violenza contro le donne e della parità di genere. Il neologismo il "femminicidio" si presenta nel significato dell'abuso sessuale e della crudeltà fisica verso le donne. Dalle osservazioni dirette, e dalla ricerca svolta sul corpus, risulta che la "violenza" sia uno degli argomenti più ricorrenti nelle campagne sociali italiane e polacche, in generale. Ciò significa che la qualità della vita è in gran parte determinata dalla presenza o dall'assenza di violenza nella vita, e la reazione agli atti di violenza è una componente importante per la definizione della responsabilità sociale 9 .

9 In entrambe le parti del corpus, la violenza non è solo associata all'abuso fisico. Il sostantivo "violenza" pol. przemoc ha un'alta frequenza in entrambe le parti del corpus: (pl. 286 types, it. 200, "violento" 17 occorrenze). Laggettivo si verifica più spesso con i sostantivi quali "compagno", "comportamento", "fidanzato", "modo" 
Tab. 3a

\begin{tabular}{|c|c|c|}
\hline Parole chiave & $\begin{array}{l}\text { Freq } \\
-\mathbf{N}^{\circ} \\
\text { types }\end{array}$ & $\begin{array}{l}\text { Definizione PL Odpowiedzialność społeczna } \\
\text { w społeczeństwie obywatelskim to: }\end{array}$ \\
\hline 1 & 2 & 3 \\
\hline $\begin{array}{l}{ }^{* *} \mathrm{CZYS}^{* *} \\
\text { EMISJA }^{* *} \\
\text { TRU(ć) }\end{array}$ & $\begin{array}{l}75 \\
25 \\
50\end{array}$ & \multirow{9}{*}{$\begin{array}{l}\text { - bycie eko }{ }^{10} \text {; } \\
\text { niezanieczyszczanie wód powierzchniowych; } \\
\text { niezaśmiecanie sieci wodociągowej; } \\
\text { OCHRONA i ratowanie zwierząt domowych } \\
\text { (pies, kot), respektowanie praw zwierząt } \\
\text { (humanitarne traktowanie np. karpi), nieporzu- } \\
\text { canie zwierząt domowych; ochrona zwierząt } \\
\text { bezdomnych; } \\
\text { - ochrona lasów i zwierząt na wolności; } \\
\text { - OSZCZEDDNOŚĆ energii elektrycznej (gasze- } \\
\text { nie światła, energooszczędne domy, żarówki, } \\
\text { oświetlenie, wyłączanie prądu pod nieobecność } \\
\text { w domu); oszczędność energii cieplnej (wy- } \\
\text { łączanie grzejników, kaloryferów); mniejsze } \\
\text { zużycie paliwa (ekojazda, przesiadanie się } \\
\text { do środków transportu, na rower); } \\
\text { oszczędzanie wody w domu (zakręcanie wody); } \\
\text { niemarnowanie jedzenia; } \\
\text { segregowanie śmieci-recykling i zagospodaro- } \\
\text { wanie odpadów, selektywna zbiórka odpadów; } \\
\text { docenianie wartości ukrytej w odpadach (pa- } \\
\text { pier, szkło, plastik, zużyte baterie); stosowanie } \\
\text { odpowiednich pojemników na różnego rodzaju } \\
\text { odpady; }\end{array}$} \\
\hline $\begin{array}{l}\text { CHRONIĆ/ } \\
\text { OCHRONA } \\
\text { OPIEKOWAĆ (SIĘ) } \\
\text { OPIEKA }\end{array}$ & $\begin{array}{l}90 / 100 \\
6 \\
44\end{array}$ & \\
\hline EKO $^{* *}$ & 70 & \\
\hline $\begin{array}{l}\text { ENERGIA + } \\
\text { ENERG }^{* *}\end{array}$ & 115 & \\
\hline KLIMAT & 58 & \\
\hline $\begin{array}{l}\text { MARNOWAĆ + } \\
\text { MARNOWANIE }\end{array}$ & 60 & \\
\hline $\begin{array}{l}\text { OSZCZĘDZAĆ + } \\
\text { OSZCZĘDNOŚĆ } \\
(\mathrm{NY})\end{array}$ & 115 & \\
\hline $\begin{array}{l}\text { PAPIER } \\
\text { PLASTIK } \\
\text { SZKLO } \\
\end{array}$ & $\begin{array}{l}32 \\
27 \\
23\end{array}$ & \\
\hline $\begin{array}{l}\text { SEGREGOWAĆ } \\
\text { + SEGREGACJA }\end{array}$ & 85 & \\
\hline
\end{tabular}

e "uomo" (tutti al maschile). Inoltre, nelle pubblicità polacche di notano i sostantivi come "cyberprzemoc" (5) e "hejt" (20), mentre in italiano "stalking" (7). Tutti si riferiscono all'abuso verbale, di solito su Internet o nella comunicazione diretta. Nella parte italiana del corpus sono presenti anche "bullo" (11) e "bullismo" (30), non verificatisi nella parte polacca. Si riferiscono principalmente alla violenza fisica e corporale, sempre nel contesto della violenza scolastica.

10 Laggettivo pol. ekologiczny si verifica più frequentemente rispetto all'ecologico italiano (55 vs 11). Nelle pubblicità polacche si trovano dei neologismi con il prefisso -eko (pol. ekonsument, ekojazda, ekoszyk, ekograty, ekoznaczenie): mentre i composti italiani sono abbastanza rari (ecolampadine, ecoastuccio, ecopunti, ecomafia). Appare anche il sostantivo prosument (pol. producent + konsument), il quale si riferisce a persone che allo stesso producono e consumano l'energia elettrica. 


\begin{tabular}{|c|c|c|}
\hline 1 & 2 & 3 \\
\hline $\begin{array}{l}\text { ŚMIECI + ŚMIECIĆ } \\
\text { (ZAŚMIECAĆ) } \\
+ \text { +ŚMIETNIK } \\
\text { ODPADY }\end{array}$ & $\begin{array}{l}200 \\
90\end{array}$ & \multirow[t]{6}{*}{$\begin{array}{l}\text { zmniejszenie EMISJI (np. spalin), niepalenie } \\
\text { śmieci, węgla w piecach grzewczych, ogranicze- } \\
\text { nie zanieczyszczeń; CZYSTA energia, czyste } \\
\text { powietrze, brak „kopciuchów”. }\end{array}$} \\
\hline ŚRODOWISKO & 125 & \\
\hline WODA & 112 & \\
\hline $\begin{array}{l}\text { (Z)UŻYCIE + } \\
\text { (Z)UŻYWAĆ } \\
\text { + UŹY Y** }\end{array}$ & 155 & \\
\hline $\begin{array}{l}\text { ZIEMIA / } \\
\text { NATURA** }^{* *}\end{array}$ & $50 / 45$ & \\
\hline $\begin{array}{l}\text { ZWIERZ** + gatunki } \\
\text { zwierząt PIES/PSI }\end{array}$ & $\begin{array}{l}85 \\
40\end{array}$ & \\
\hline
\end{tabular}

Fonte: elaborazione propria

Tab. $3 b$

\begin{tabular}{|c|c|c|}
\hline Parole chiave & $\begin{array}{l}\text { Freq } \\
-\mathbf{N}^{\circ} \\
\text { types }\end{array}$ & $\begin{array}{l}\text { Definizione IT La responsabilità sociale/ } \\
\text { senso civico nella società civile è: }\end{array}$ \\
\hline 1 & 2 & 3 \\
\hline \begin{tabular}{|l|} 
PULI \\
INQUINA**
\end{tabular} & $\begin{array}{l}80 \\
30 \\
\end{array}$ & \multirow{6}{*}{$\begin{array}{l}\text { - ambiente pulito; riduzione di inquinamento } \\
\text { strade, città e mare senza rifiuti, mozziconi } \\
\text { e deiezioni canine; } \\
\text { DIFESA del verde; } \\
\text { - energia pulita e creativa, risparmio energetico; } \\
\text { non sprecare energia; uso e consumo } \\
\text { responsabile di energia elettrica; energie } \\
\text { rinnovabili; } \\
\text { NON ABBANDONARE gli animali } \\
\text { domestici prima delle vacanze; lotta contro il } \\
\text { maltrattamento degli animali; } \\
\text { raccolta DIFFERENZIATA dei rifiuti } \\
\text { (DIFFERENZIARE); fare la differenza } \\
\text { riciclare, dare una nuova vita ai rifiuti; gestion } \\
\text { dei rifiuti; }\end{array}$} \\
\hline $\begin{array}{l}\text { DIFENDERE } \\
\text { + DIFESA } \\
\text { PROTEGGERE } \\
\text { + PROTEZIONE } \\
\text { TUTELARE } \\
\text { + TUTELA } \\
\end{array}$ & 67 & \\
\hline ECO $^{* *}$ & 15 & \\
\hline $\begin{array}{l}\text { ENERGIA + } \\
\text { ENERG** }\end{array}$ & 140 & \\
\hline CLIMA** $^{* *}$ & 10 & \\
\hline $\begin{array}{l}\text { SPRECARE } \\
+ \text { SPRECO }\end{array}$ & 98 & \\
\hline
\end{tabular}

11 Nelle pubblicità italiane il sostantivo "inquinamento" appare anche nel significato di inquinamento acustico. 
Tab. 3b (cont.)

\begin{tabular}{|c|c|c|}
\hline 1 & 2 & 3 \\
\hline $\begin{array}{l}\text { CARTA /CARTONE } \\
\text { PLASTICA } \\
\text { VETRO }\end{array}$ & $\begin{array}{l}150 \\
196 \\
115\end{array}$ & \multirow{8}{*}{$\begin{array}{l}\text { RISPARMIO dell'acqua potabile (idrico), } \\
\text { lotta contro lo spreco dell'acqua, usare; uguale } \\
\text { accesso all'acqua; } \\
\text { tutela dei diritti degli animali; abolizione della } \\
\text { caccia e degli esperimenti sugli animali. }\end{array}$} \\
\hline $\begin{array}{l}\text { DIFFERENZIARE } \\
+ \text { DIFFERENZIATA } \\
+ \text { +**DIFFER }{ }^{* *} \\
\text { DIFFERENZ }\end{array}$ & 350 & \\
\hline $\begin{array}{l}\text { RIFIUTO } \\
\text { + RIFIUTARE }\end{array}$ & 310 & \\
\hline AMBIENTE - 169 & 169 & \\
\hline ACQUA & 443 & \\
\hline CONSUMO (-ARE) & 117 & \\
\hline $\begin{array}{l}\text { TERRA } \\
\text { NATURA } \\
\text { VERDE }\end{array}$ & $\begin{array}{l}30 \\
50 \\
45\end{array}$ & \\
\hline $\begin{array}{l}\text { ANIMAL } \\
\text { + gatunki zwierząt } \\
\text { CANE + CANi }\end{array}$ & $\begin{array}{l}230 \\
120\end{array}$ & \\
\hline
\end{tabular}

Fonte: elaborazione propria

\section{Conclusioni}

Sulla base delle sei tabelle si può desumere che gli esponenti lessicali analizzati sono generalmente simili tra la parte italiana e polacca del corpus raccolto. Tuttavia, vale la pena sottolinear che i frames presentati non solo non sono eterogenei, ma prima di tutto sono dinamici. I loro componenti cambiano con lo sviluppo direzionale e tematico del discorso della pubblicità sociale. Pertanto, le proporzioni tra le occorrenze delle singole parole chiave possono variare, se si applica la chiave diacronica nell'analisi lessicale ${ }^{12}$.

12 Ad esempio, nel 2019 si è potuto osservare un aumento del numero di annunci polacchi dedicati alla descrizione e alla spiegazione molto accurata della corretta raccolta dei rifiuti, e alla partecipazione nelle elezioni. In Italia, invece, si registra un proliferare delle pubblicità relative ai pregiudizi nazionali, razziali e quelle dedicate all'integrazione (principalmente di immigrati), seguite dagli annunci contro lo spreco di energia, acqua e cibo (ad esempio \#IntegrAction2019; Ci riesco SQUAD). 
Dopo aver analizzato i testi raccolti fino al 2017, si può comunque affermare che nelle pubblicità italiane in quest'area tematica, il problema delle pari opportunità tra donne e uomini, e la relativa ad essi violenza contro le donne, è discusso più spesso che nella parte polacca. L'argomento dei migranti è presente soltanto nella parte italiana del corpus, similmente al problema del razzismo. Il tema del volontariato, dell'assistenza e dell'adozione "a distanza", ovvero il sostegno ai bambini delle aree povere e minacciate, è anche molto più presente nella parte italiana. Nelle pubblicità polacche invece prevale la coppia sostantivo e aggettivo pol. niepetnosprawność e niepetnosprawny (oltre 120 types), le quali si riflettono nel numero più alto delle pubblicità legate al supporto e ai pari diritti per persone con disabilità. Qualche argomento, come quello dell'affluenza (il voto) e l'interesse per le elezioni come un esponente di un atteggiamento pro-sociale non appare affatto nel discorso sociale italiano. Per di più non si notano le pubblicità dedicate alla vendita dell'alcol ai minori.

Nel contesto della tutela ambientale, vi sono alcune differenze tra gli esponenti del concetto di responsabilità sociale e quindi tra le definizioni elaborate. La prima riguarda una significativa frequenza del sostantivo "acqua" e dei messaggi pubblicitari italiani dedicati al consumo, allo spreco e al risparmio delle risorse idriche. Nella parte polacca del corpus il sostantivo pol. woda ha un moderato numero di occorrenze (100), mentre nella parte italiana questo numero è oltre quattro volte superiore (430). Questa disproporzione è causata non solo dalle condizioni climatiche nella penisola appenninica, ma anche da una diversa valutazione dell'importanza e da un'approfondita valorizzazione dell'acqua in Italia (in particolare acqua potabile, ma anche e soprattutto dell'acqua marina) e del suo contributo alla qualità della vita. L'acqua nel DPS italiano è un sinonimo della vita, è un bene comune le cui risorse vengono percepite come limitate nella coscienza sociale. Lacqua italiana diventa un valore in sé ed il suo risparmio è un investimento per una migliore qualità della vita.

La questione più comunemente accennata nel contesto della tutela ambientale nel DPS italiano è il problema dell'ordine, dello smaltimento e del riciclaggio dei rifiuti. Molto spazio è dedicato all'argomento anche nelle pubblicità polacche, ma si nota una significativa prevalenza degli esponenti italiani. Questa osservazione si applica sia alle parole "differenziare", "differenziata" (raccolta), "differenza", utilizzate solo nel contesto della spazzatura (>400 types), ma anche alla prevalenza dei sostantivi italiani che denominano i materiali sottoposti al recycling ${ }^{13}$. Alcune differenze importanti

13 In totale 1160 types nella parte italiana e 420 in quella polacca. Anche in questo caso, vale la pena di sottolineare che in Polonia nell'anno 2019 sono state lanciate le 
si possono osservare anche nel caso della protezione degli animali, essa intesa come uno degli elementi di protezione ambientale. Si notano più pubblicità italiane incentrate sul problema dell'abbandono (alta frequenza di "abbandonare" e "abbandono") degli animali domestici, in particolare cani e gatti. Gli esponenti polacchi della responsabilità sociale in materia della tutela ambientale sembrano più generici, a volte riguardano la cura della fauna selvatica, dei boschi. Tuttavia, si menzionano più specie di animali.

Si è detto che il concetto della società civile si riflette prima di tutto in un alto livello di autocoscienza collettiva-cognitiva e normativa. I suoi principi costruiscono una sorta di visione del mondo ed influenzano la disposizione all'azione. L'accettazione dell'impegno di agire per il bene comune è importante al momento di prendere decisioni riguardanti interessi o ideali contrastanti, nonché di risolvere questioni delicate, legate, ad esempio, alla tolleranza verso la diversità. Ciò aiuta a mantenere un equilibrio tra i componenti concorrenti o contraddittori all'interno della società e consente alla comunità di funzionare nel suo insieme.

La "società civile" è un'espressione comunemente utilizzata, e la realizzazione degli ideali che ne formano le fondamenta è diventata uno degli obiettivi della politica sociale dell'Unione europea. Il termine stesso, tuttavia, è ambiguo e talvolta prende in considerazione solo le misure apolitiche, oppure abbraccia anche la partecipazione alla vita politica, o ilvoto nelle elezioni. Imedia svolgono un ruolo importante nella definizione del concetto, in particolar modo la pubblicità non commerciale. In Italia, nonostante siano trascorsi molti anni dall'Unità d'Italia e anche in Polonia, a 30 anni dalla caduta del comunismo, il significato della nozione: "responsabilità sociale" ed il suo valore non è del tutto trasparente per nessuna delle due nazioni. I frames analizzati non hanno i componenti ben precisi, i punti di riferimento chiari per tutta la società. Sia in Polonia che in Italia il bene comune ed il bene degli altri (delle minoranze) vengono spesso trascurati ed il mancato senso della responsabilità collettiva, ad esempio per i disastrosi cambiamenti climatici, deriva dall'ignoranza o dall'incomprensione dei problemi sociali. Si sottovaluta la responsabilità di tutti i cittadini per la qualità della vita in una società integrata. La chiusura ai problemi, o semplicemente una loro insufficiente comprensione, possono, almeno in una certa misura, essere superate grazie al discorso della pubblicità sociale. Essa, attraverso i suoi messaggi, indica, definisce e spiega i fattori che hanno un impatto significativo sul deterioramento della qualità della vita e sul degrado della società integrata.

pubblicità dedicate ad una corretta e molto precisa segregazione dell'immondizia. Ciò è dovuto all'introduzione delle restrizioni legali nel campo della gestione dei rifiuti. 


\section{Bibliografia}

Cartocci, Roberto (2007). Mappe del tesoro. Atlante del capitale sociale in Italia, Bologna, il Mulino, pp. 103-107.

Croft, William (2002). Cognitive Linguistics, Cambridge, Cambridge University Press.

Fillmore, Charles (1982). "Frame Semantics", [in] John R. Taylor (a c. di), 2006, Cognitive Linguistics Research, 34, De Gruyter, pp. 373-400.

Fillmore, Charles (1985). "Frames and The Semantics of Understanding", Quaderni di Semantica, 6 (2), Bologna, il Mulino, pp. 222-254.

Gleason, Jean Berko e Ratner, Nan Bernstein (2005). Psycholingwistyka, Gdańsk, GWP.

Krasnowolski, Andrzej (2014). Społeczeństwo obywatelskie i jego instytucje, OT-627, Warszawa, Biuro Analiz Dokumentacji, Kancelaria Senatu RP.

Putnam, Robert D. (1993). La tradizione civica nelle regioni italiane, Milano, Mondadori, pp. 73-96 e pp. 176-190.

Załęski, Paweł (2012). Neoliberalizm i społeczeństwo obywatelskie, Toruń, WUMK. 